\title{
COMPREENSÃO DE LEITURA E CONSCIÊNCIA MORFOLÓGICA EM CRIANÇAS DO ENSINO FUNDAMENTAL
}

Patrícia Silva Lúcio

Universidade Estadual de Londrina

Thatiana Helena de Lima

Universidade Federal da Bahia

\section{Ana Deyvis Santos Araújo Jesuíno}

Universidade São Francisco

Fabián J. M. Rueda

Universidade São Francisco

\begin{abstract}
Resumo
O objetivo do estudo foi investigar os efeitos da idade e escolaridade no desempenho em compreensão leitora e consciência morfológica, assim como o poder preditivo da consciência morfológica no desempenho em compreensão leitora. Participaram 403 estudantes do $2^{\circ}$ ao 90 ano do Ensino Fundamental (Ciclos 1 e 2) de uma cidade do interior do Estado do Paraná ( $54,3 \%$ do sexo feminino). Como instrumentos, foram utilizados o Teste de Cloze (texto "Uma vingança infeliz") e Tarefas de Avaliação da Consciência Morfológica. Os resultados mostraram que tanto a compreensão em leitura quanto a consciência morfológica sofrem influência desenvolvimental e que a consciência morfológica pode predizer entre $23 \%$ e $34 \%$ da compreensão leitora.
\end{abstract}

Palavras-chave: teste de Cloze; compreensão leitora; leitura; consciência morfológica; predição.

\section{READING COMPREHENSION AND MORPHOLOGICAL AWARENESS IN ELEMENTARY SCHOOL CHILDREN}

\begin{abstract}
The aim of the study was to investigate the effects of age and schooling in performance in reading comprehension and morphological awareness, as well as the predictive power of morphological awareness in reading comprehension. Students $(n=403)$ from 2 nd to 9th year of Elementary School (cycles 1 and 2) of a city in the state of Paraná participated of the study $(54.3 \%$ female). As instruments, the Cloze Test (text "Unfortunate revenge") and Morphological Consciousness Assessment Tasks were administered. The results showed that both reading comprehension and morphological awareness have a developmental influence, and that morphological awareness can predict between $23 \%$ and $34 \%$ of reading comprehension.

Keywords: Cloze test; reading comprehension; reading; morphological awareness; prediction.
\end{abstract}




\title{
COMPRENSIÓN LECTORA Y CONCIENCIA MORFOLÓGICA EN NIÑOS DE ENSEÑANZA PRIMARIA
}

\begin{abstract}
Resumen
El objetivo del estudio fue investigar los efectos de la edad y de la enseñanza en el desempeño de la comprensión en lectura y en la consciencia morfológica, así como también el poder que la consciencia morfológica tiene para predecir el desempeño en comprensión lectora. Participaron 403 estudiantes del $2^{\circ}$ al $9^{\circ}$ grado de la Enseñanza (10 Ciclo) Primaria de una ciudad del interior del Estado de Paraná (54,3\% niñas). Fueron utilizados como instrumentos el Test de Cloze (texto "Una venganza infeliz") y Tareas de Evaluación de la Consciencia Morfológica. Los resultados mostraron que tanto la comprensión lectora como la consciencia morfológica sufren influencia del desarrollo, y que la consciencia morfológica puede predecir entre $23 \%$ y $34 \%$ de la comprensión lectora.
\end{abstract}

Palabras clave: test de Cloze; comprensión lectora; lectura; consciencia morfológica; predicción.

\section{INTRODUÇÃO}

Em um mundo letrado, ler e compreender o que foi lido representam importantes habilidades que contribuem para a adaptação e sobrevivência, uma vez que se lê para pegar um ônibus, para utilizar o caixa eletrônico, para fazer compras, por prazer, dentre muitas outras possibilidades. Nesse sentido, ao estar imerso em uma sociedade em que os processos de leitura fazem parte do cotidiano, o surgimento de dificuldades de compreensão de textos durante a aquisição dessa habilidade constitui um problema que preocupa a pais, professores e entidades educacionais.

A leitura é considerada uma habilidade complexa que engloba a decodificação de um registro escrito e a análise reflexiva do conteúdo, fatores estes que conferem ao indivíduo a capacidade de compreensão, habilidade considerada fundamental para a comunicação básica entre as pessoas (Braibant, 1997). Tal processo permite a análise de situações e tomada de decisão que possibilitam uma compreensão mais ampla do mundo (Soares, 1995).

O estudo da compreensão de textos tomou impulso a partir da década de 1970, fruto do desenvolvimento das investigações sobre a linguagem, resultantes da revolução cognitiva da segunda metade do século XX (Lencastre, 2003). Desde então, diversas pesquisas têm sido conduzidas em vários países com o objetivo de conhecer os processos cognitivos, contextuais e linguísticos envolvidos na compreensão, criando modelos explicativos para esses processos (p. ex. Anglat, 2008; Keshavarz \& Salimi, 2007).

A compreensão de textos constitui a obtenção de significados e a construção de uma representação mental (Kintsch, 1988) do conteúdo lido a partir da retirada, pelo leitor, de elementos do texto e de seu próprio conhecimento de mundo (Yuill \& Oakhill, 1996). Constitui mecanismo complexo que requer o envolvimento simultâneo de diferentes habilidades linguísticas e processos cognitivos, tais como memória de trabalho, decodificação, vocabulário e capacidade de realizar 
inferências (Cain, Bryant, \& Oakhill, 2004; Freed, Hamilton, \& Long, 2017; Liu et al., 2018; Nouwens, Groen, \& Verhoeven, 2017).

A compreensão de leitura está associada de maneira direta ao processo de ensino e aprendizagem, sendo que dificuldades nesta área podem gerar prejuízos em outras áreas do desempenho do indivíduo. Por exemplo, um déficit na compreensão de leitura pode ocasionar comprometimento ao indivíduo em disciplinas que exigem a interpretação por parte do aluno (como língua portuguesa, matemática, história e geografia) e a assimilação do que foi lido, contribuindo para o baixo rendimento escolar (Cunha \& Capellini, 2016).

Um dos recursos mais utilizados para avaliar a compreensão leitora é o teste de Cloze, que tem como base a técnica criada por Taylor (1953) e que teve seus estudos iniciais no Brasil realizados por Santos (2005). Consiste em uma adaptação de textos curtos (frequentemente entre duzentas e quatrocentas palavras) que tem o enésimo vocábulo omitido (geralmente o quinto) e substituído por um traço de tamanho equivalente ao da palavra retirada (Greene, 2001; Kobayashi, 2002). Desde sua criação, os testes gerados a partir da técnica original de Cloze têm passado por vários estudos de validação como uma medida da compreensão da leitura (p. ex., Fuchs, Fuchs, \& Maxwell, 1988; Greene, 2001).

A compreensão de leitura, avaliada pelo Cloze, aparece na literatura associada a outros aspectos que influenciam a aprendizagem. Podem ser citados o desenvolvimento do desempenho ortográfico (Guimarães, Paula, Mota, \& Barbosa, 2014), consciência morfossintática (Mota et al., 2009), inteligência nãoverbal e desempenho escolar (Freitas Junior \& Mota, 2015), consciência fonológica (Suehiro \& Santos, 2015) e consciência morfológica (Mota, et al., 2009; Nagy, Berninger, \& Abbot, 2006).

A consciência morfológica é um dos facilitadores destacados na literatura para a aquisição da leitura e da escrita (Deacon \& Kirby, 2004; Nagy et al., 2006), além de fazer parte de um conjunto de habilidades metalinguísticas consideradas importantes para a alfabetização em diferentes línguas, tanto alfabéticas (p. ex., Mota et al., 2009; Tong, Deacon, Parrila, \& Kirby, 2011) quanto não alfabéticas (Ho \& Bryant, 1997; Li, Anderson, Nagy, \& Zhang, 2002). Trata-se da capacidade do indivíduo de refletir de forma consciente sobre de como as palavras podem ser divididas em unidades menores (Carlisle, 2000). Os morfemas são as menores unidades linguísticas que encerram significado (Déjean, 1998; Harris, 1955), podendo ser classificados como raízes (quando se confundem com palavras, como "feliz"); afixos (i.e., prefixos e sufixos, ou termos que modificam o radical, p.ex., infeliz); derivacionais (que mudam o significado semântico do radical, p. ex., "feliz" é um adjetivo enquanto "felicidade" é um substantivo); flexionais (que modificam o tempo verbal, o modo, o número, etc., como no português o <s $>$ em final de palavra formando o plural, p.ex., "felizes").

Apesar de menos investigada do que a consciência fonológica, é bem documentada na literatura a relação entre a consciência morfológica e a habilidade 
de compreensão leitora. Tais estudos têm sido conduzidos com amostras de crianças e adultos e subpopulações específicas, como de pessoas com dislexia. Em geral, os estudos têm demonstrado que: a consciência morfológica contribui de forma única para o desempenho em compreensão; pode estar alterada em grupos de disléxicos ou de pessoas com problemas de aprendizagem; e o uso de programas de treinamento pode proporcionar resultados melhores em compreensão leitora (p. ex., Diamanti et al., 2017; Law, Wouters, \& Ghesquière, 2015; Li, Dronjic et al., 2017; Lyster, Lervåg, \& Hulme, 2016; Manolitsis, Grigorakis, \& Georgiou, 2017; Tighe \& Schatschneider, 2016b). Corroborando esses achados, uma meta análise recente (Tighe \& Schatschneider, 2016a) com 16 estudos independentes (variação etária dos 16 aos 83 anos) investigou a contribuição de 10 habilidades (tais como vocabulário oral e decodificação) no desempenho em compreensão leitora. Foi demonstrado que seis variáveis apresentaram contribuições fortes para a compreensão $(>0,50)$, sendo a consciência morfológica uma delas (média 0,59; variação: 0,47-0,68).

Pesquisadores brasileiros também têm se interessado pelo tema da relação entre a consciência morfológica e os processos de leitura. Mota, Anibal e Lima (2008) estudaram crianças de $1^{a}$ e $2^{a}$ série em um conjunto de tarefas de consciência morfológica e sua relação com a consciência fonológica, a leitura e a escrita. As autoras demonstraram que a consciência morfológica derivacional contribui de forma independente da idade e da consciência fonológica para a leitura e escrita das crianças avaliadas. Nesta mesma linha, Mota et al. (2009) observaram correlações significativas entre o desempenho em tarefas de consciência morfológica e o teste de Cloze entre crianças de $1^{a}$ e $2^{a}$ séries, mas estas correlações perderam significância entre as mais jovens após controle da consciência fonológica.

Em outro estudo realizado, Guimarães et al. (2014) verificaram as relações entre consciência morfológica, ortografia e compreensão de leitura. As crianças do estudo ( $3^{\circ}$ ao $5^{\circ}$ ano) foram separadas em dois grupos de acordo com 0 desempenho ortográfico (baixo e alto). Os resultados indicaram que os alunos com baixo desempenho ortográfico também apresentaram um desempenho inferior em compreensão de leitura e consciência morfológica. A análise de regressão evidenciou que o desempenho nas tarefas de consciência morfológica (flexional e derivacional) contribuiu de forma significativa para explicar a variância na compreensão de leitura.

Tendo em vista a importância da consciência morfológica para os processos de alfabetização e desempenho em leitura e escrita (Mota, 2009), assim como da importância da avaliação de diferentes habilidades metalinguísticas para se investigar possíveis dificuldades em habilidades de ordem superior (como a compreensão da leitura) e traçar planos diagnósticos e interventivos (Spinillo, Mota, \& Correa, 2010); esta pesquisa teve por objetivos: (1) descrever o desempenho de crianças dos Ciclos 1 e 2 ano do Ensino Fundamental no teste de 
Cloze e em tarefas de consciência morfológica, investigando os efeitos da idade e do ciclo escolar nesses construtos; (2) investigar a correlação entre estas habilidades e o poder preditivo da consciência morfológica no desempenho em compreensão leitora. Conforme apresentado nesta introdução, estudos têm demonstrado que a consciência morfológica contribui de forma única para a compreensão leitora, para além do controle de variáveis tradicionais como a decodificação e a consciência fonológica. Além disso, esses resultados têm sido encontrado em uma pluralidade de amostras (i.e., população específica ou não) e faixas etárias, o que reforça a importância desta variável para os estudos em compreensão leitora. Neste sentido, o estudo busca fornecer resultados que clarifiquem a relação entre a consciência morfológica e a compreensão leitora em uma amostra de crianças de diferentes níveis de desenvolvimento (Ciclos 1 e 2 do ensino fundamental).

\section{MÉTODO}

\section{Participantes}

Esta pesquisa faz parte de um projeto maior que visa buscar evidências de validade em múltiplas tarefas relacionadas ao desempenho escolar entre crianças e adolescentes do $2^{\circ}$ ao 90 ano do Ensino Fundamental. A amostra coletada foi de 403 estudantes de escolas municipais $(60,8 \%)$ e estaduais $(39,2 \%)$ de uma cidade do interior do Estado do Paraná ( $n=45,44,56,100,59,69,17$ e 12 para alunos do 20 ao 90 ano, respectivamente; um estudante com dado ausente). A maioria das crianças era do sexo feminino (54,3\%) e a média de idade foi de 10,52 anos ( $D P=1,77$; mínimo 6 e máximo 15 anos). Os alunos foram avaliados em diversos instrumentos, sendo que, para os propósitos do presente estudo, foram utilizados apenas os dados referentes ao teste de Cloze e da tarefa de Consciência Morfológica. As mesmas tarefas foram utilizadas em todos os anos, de modo a possibilitar comparações na mesma escala de medida (Urbina, 2014). Um total de 388 participantes completaram o teste de Cloze e 138 estudantes completaram a tarefa de consciência morfológica.

\section{Instrumentos}

Teste de Cloze - texto "Uma vingança infeliz" (Santos, 2005)

Com o objetivo de avaliar a compreensão de leitura, o teste é composto por um texto com 102 vocábulos, em que, na sua forma tradicional, são omitidos os quintos vocábulos. As palavras são substituídas por um traço correspondente ao tamanho da palavra. O avaliando deve ler silenciosamente o texto até o final e, após, retornar a leitura completando os espaços com as palavras que julga dar sentido ao texto. A correção utilizada é a literal, considerando apenas as palavras idênticas às retiradas do texto. A pontuação máxima é de 15 pontos. Diversos 
estudos apontam o instrumento como uma medida válida da compreensão de leitura, com adequados índices psicométricos de precisão (alfa de Cronbach 0,83; Santos, 2005), validade de critério por grupo contrastante (Santos \& Oliveira, 2010) e validade por processo de resposta (Cunha \& Santos, 2009).

Tarefas de Avaliação da Consciência Morfológica - TCM (Guimarães \& Mota, 2016, adaptadas de Nunes, Bindman \& Bryant, 1997)

O instrumento é composto de duas séries de tarefas, são elas: (a) Analogia derivacional, com 17 itens, perante os quais a criança deverá ser capaz de formar uma palavra morfologicamente complexa relacionada à palavra alvo (p. ex.: tênistenista; arte; ?); (b) Analogia flexional, contendo 19 itens, referentes à concordância verbal, à flexão de número e à flexão de gênero (p. ex., "Andaandou; olha?). A resposta correta exige que a criança identifique a modificação morfossintática em um par de palavras, para em seguida aplicá-la em outro par. A relação morfossintática entre as palavras deve ser mantida pelas crianças. O escore na tarefa pode ser dado pela soma dos itens relativos à analogia derivacional, analogia flexional e por escore total (escore máximo = 36 pontos). Estudo de validade e precisão pode ser observado em Mota, Santos e Guimarães (2014). As autoras demonstraram efeito de série para a morfologia derivacional e para o escore total na tarefa, mas não para a morfologia flexional. A correlação das tarefas entre si foi significativa e também destas com a consciência fonológica. A análise pelo alfa de Cronbach mostrou resultados abaixo do esperado em termos de consistência interna para as três medidas (respectivamente, 0,59, 0,61 e 0,66 para a morfologia derivacional, flexional e escore total, respectivamente).

\section{Procedimentos}

Depois da autorização das escolas, o projeto foi encaminhado e aprovado pelo Comitê de Ética da instituição de origem (CAAE: 59601916.0.0000.5231). Depois da sua aprovação, as escolas foram novamente contatadas e a aplicação foi realizada na própria escola, com as crianças cujos pais autorizaram por escrito por meio do Termo de Consentimento Livre e Esclarecido. Os testes tiveram aplicação individual ou coletiva, em sala de aula fornecida pela escola. Para efeito desta pesquisa, informa-se que o teste de Cloze foi aplicado de forma coletiva e o de consciência morfológica individualmente. O procedimento durou, aproximadamente, 30 minutos na aplicação coletiva e 20 minutos na individual.

\section{Análise dos dados}

Primeiramente serão apresentadas as análises descritivas a partir das pontuações obtidas pelos participantes nos dois instrumentos e análise de consistência interna das tarefas. Posteriormente foram realizadas estatísticas inferenciais, utilizando a comparação entre grupos (análises univariadas de variância), correlações (Pearson e correlação parcial) e análise de regressão linear. 
Os dados foram analisados por meio do programa estatístico SPSS for Windows, versão 20.0. Para que as análises sejam estatisticamente significativas, utilizouse como critério o valor de $p \leq 0,05$. Optou-se por utilizar estatísticas paramétricas a despeito da ausência de normalidade dos dados em função do tamanho amostral do estudo, i.e., em amostras maiores ( $n>40$ ) a violação de normalidade não constitui um problema (p. ex., Elliott \& Woodward, 2007; Ghasemi \& Zahediasl, 2012; Pallant, 2010). Resultados para teste kolmogorov-Smirnov (que testa a hipótese nula de que a distribuição é normal) foi de $\mathrm{p}<0,001$ para todas as tarefas de desfecho utilizadas.

\section{RESULTADOS}

A Tabela 1 apresenta as estatísticas descritivas do teste de Cloze para a amostra geral e por sexo e ano escolar. Para esta última variável, dois grupos foram criados, correspondentes ao primeiro e segundo ciclos do ensino fundamental. Este procedimento foi adotado devido a diferenças no tamanho amostral, com os anos finais apresentando $n$ amostral relativamente menor, especialmente $8^{\circ}$ e $9^{\circ}$ anos (ver seção participantes). Assim, no primeiro grupo ficaram os alunos do $2^{\circ}$ ao $5^{\circ}$ ano e no segundo ficaram os do $6^{\circ}$ ao $9^{\circ}$ ano. Desse modo, observa-se que os estudantes obtiveram pontuação mínima no instrumento de compreensão de leitura, mas não a máxima. Os índices de consistência interna (alfa de Cronbach) foram considerados adequados, todos acima de 0,70. A exceção ficou por conta do Ciclo 2, que atingiu valor abaixo do esperado.

Tabela 1.

Estatística descritiva no instrumento Cloze para a amostra geral e separada por sexo e escolaridade (Ciclo 1 e Ciclo 2)

\begin{tabular}{lcccccc}
\hline Amostra & $N$ & Média & DP & Mínimo & Máximo & $\alpha$ \\
\hline Geral & 388 & 6,53 & 3,08 & 0,00 & 13,00 & 0,70 \\
Feminino & 176 & 6,61 & 3,12 & 0,00 & 13,00 & 0,69 \\
Masculino & 212 & 6,43 & 3,03 & 0,00 & 12,00 & 0,70 \\
Ciclo 1 & 231 & 5,27 & 3,04 & 0,00 & 13,00 & 0,74 \\
Ciclo 2 & 157 & 8,38 & 2,03 & 0,00 & 13,00 & 0,54 \\
\hline
\end{tabular}

Nota. O escore máximo na tarefa é de 15 pontos.

Uma análise univariada de variância (ANOVA) foi conduzida para observar a presença de efeito geral nos escores do teste de Cloze, utilizando-se como fatores fixos o sexo e o ciclo. Houve um efeito geral entre sujeitos nos escores ( $F(3$, $384)=43,34, p<0,001, \eta 2=0,253)$. O modelo total apresentou um $R^{2}$ ajustado de 0,247 (i.e., explicou $24,7 \%$ da variância total). Também foram observadas diferenças entre os ciclos escolares e, conforme a Tabela 1, no Ciclo 2 as médias 
no teste foram mais elevadas $\left(F(1,384)=129,53, p<0,001, \eta^{2}=0,252\right)$. No entanto, não houve diferenças de sexo nos escores $(F(1,384)=2,00, p=0,158$, $\left.\eta^{2}=0,005\right)$, e tampouco foi observada interação do sexo com o Ciclo ( $F(1$, $\left.384)=0,80, p=0,373, \eta^{2}=0,002\right)$. A Figura 1 ilustra as diferenças de desempenho dos participantes no teste de Cloze nos Ciclos 1 e 2 e por sexo. Observa-se que há sobreposição em todos os anos escolares dos escores dos meninos e das meninas, mas a distribuição dos escores no Ciclo 1 tende a se concentrar mais na porção esquerda do gráfico (escores mais baixos), enquanto que no Ciclo 2 os escores tendem a se concentrar na parte direita (escores mais elevados), conforme demonstrado pelo resultado da ANOVA.

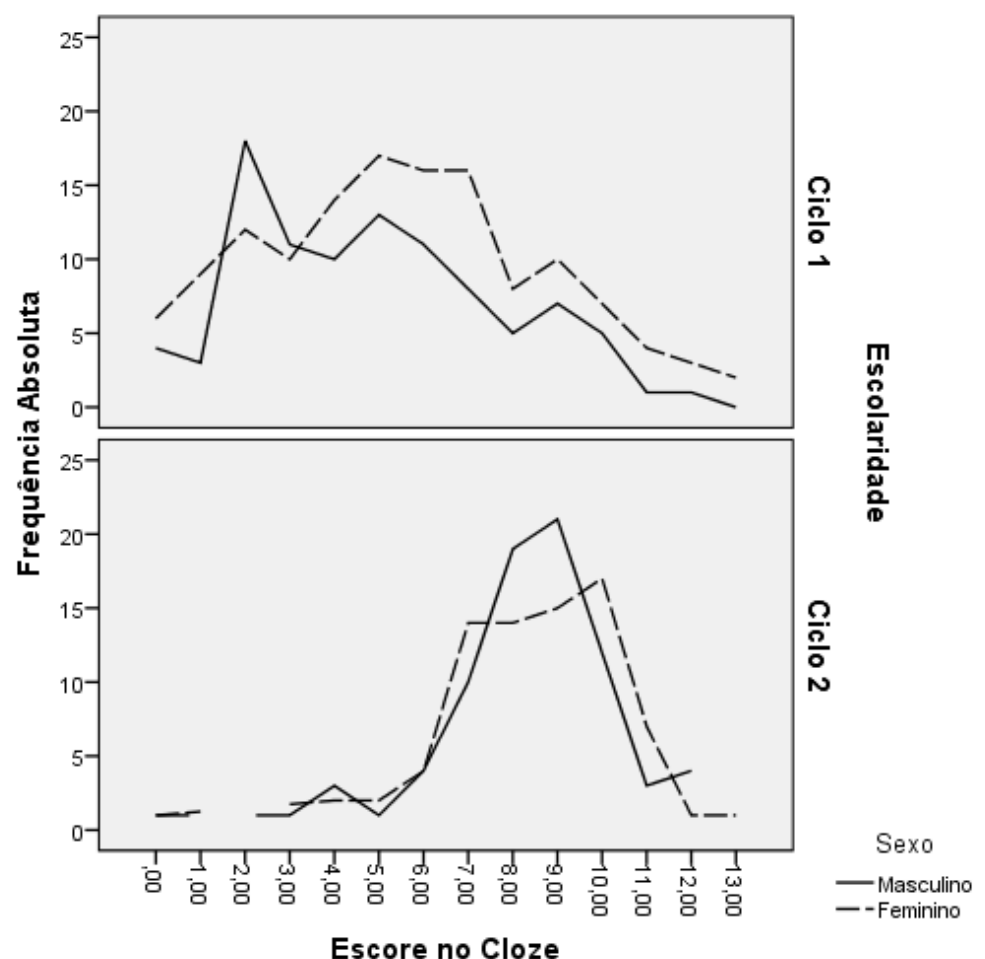

Figura 1. Distribuição dos escores no Teste de Cloze em função do sexo e do ano escolar (Ciclos 1 e 2).

A Tabela 2 apresenta as estatísticas descritivas para a amostra geral, por sexo e por ciclo para a tarefa de consciência morfológica. Para esta tarefa, os escores também foram divididos pelo tipo de consciência morfológica avaliada (derivacional, flexional e escore geral). Conforme se observa, nenhum participante obteve escore mínimo, mas o escore máximo foi obtido em subtipos das tarefas (apesar de o escore máximo no total não ter ocorrido). A consistência interna das tarefas e por subgrupo foi em geral adequada, com índices abaixo do esperado para o Ciclo 2 (derivacional e flexional) e para o sexo masculino na tarefa flexional. 
Tabela 2.

Estatística descritiva para a TCM (derivacional, flexional e escore total) para a amostra geral e separada por sexo e escolaridade (Ciclo 1 e Ciclo 2)

\begin{tabular}{cccccccc}
\hline TCM & Amostra & $N$ & Média & DP & Mínimo & Máximo & $\alpha$ \\
\hline \multirow{5}{*}{ Derivacional } & Geral & 138 & 11,07 & 3,01 & 3 & 17 & 0,74 \\
& Feminino & 74 & 11,01 & 2,95 & 3 & 16 & 0,71 \\
& Masculino & 64 & 11,14 & 3,51 & 4 & 17 & 0,79 \\
& Ciclo 1 & 80 & 9,90 & 3,19 & 3 & 16 & 0,72 \\
& Ciclo 2 & 58 & 12,69 & 2,47 & 6 & 17 & 0,63 \\
\hline \multirow{5}{*}{ Flexional } & Geral & 138 & 12,53 & 3,50 & 1 & 19 & 0,71 \\
& Feminino & 74 & 12,62 & 3,44 & 1 & 18 & 0,76 \\
& Masculino & 64 & 12,44 & 3,58 & 4 & 19 & 0,67 \\
& Ciclo 1 & 80 & 11,55 & 3,75 & 1 & 19 & 0,70 \\
& Ciclo 2 & 58 & 13,90 & 2,58 & 5 & 18 & 0,60 \\
\hline \multirow{5}{*}{ Total } & Geral & 138 & 23,61 & 5,87 & 5 & 33 & 0,82 \\
& Feminino & 74 & 23,64 & 5,60 & 5 & 33 & 0,83 \\
& Masculino & 64 & 23,58 & 6,21 & 8 & 33 & 0,81 \\
& Ciclo 1 & 80 & 21,45 & 6,00 & 5 & 33 & 0,79 \\
& Ciclo 2 & 58 & 26,59 & 4,17 & 15 & 33 & 0,72 \\
\hline
\end{tabular}

Nota. O escore máximo nas tarefas: derivacional $=17$ pontos; flexional $=19$ pontos; escore total $=36$ pontos.

Novamente, análises univariadas de variância com o sexo e o Ciclo como fatores fixos foram conduzidas. Análises separadas para os subtipos e para o escore total na tarefa foram avaliados. O mesmo padrão foi encontrado nas três análises. Desse modo, um efeito geral foi obtido em todas as comparações ( $F(3$, $134)=10,34, p<0,001, \eta^{2}=0,188$ para a TCM derivacional; $F(3,134)=5,82$, $\mathrm{p}<0,001, \eta^{2}=0,115$ para a TCM flexional e $F(3,134)=10,34, p<0,001, \eta^{2}=0,188$ para o escore total). O modelo total explicou, respectivamente, $18,8 \%, 9,5 \%$ e $17 \%$ da variância ( $R^{2}$ ajustado). Observou-se que o efeito obtido deve-se às diferenças entre os estudantes dos Ciclos 1 e 2 e, pela Tabela 2, pode-se perceber que os estudantes que estão anos escolares mais avançados obtiveram médias significativamente maiores quando comparados aos dos anos primários $(F(1$, $134)=30,27, p<0,001, \eta^{2}=0,187$ para a TCM derivacional; $F(1,134)=16,22$; $\mathrm{p}<0,001, \mathrm{n}^{2}=0,108$ para a TCM flexional e $\mathrm{F}(1,134)=30,76, \mathrm{p}<0,001, \mathrm{\eta}^{2}=0,197$ para o escore total). Não houve diferenças de sexo $(F(1,134)=0,12, p=0,734$, $\eta^{2}=0,001$ para a TCM derivacional; $F(3,134)=0,21, p=0,649, \eta^{2}=0,002$ para a TCM flexional e $F(1,134)=0,01, p=0,923, \eta^{2}=0,000$ para o escore total) e nem interação sexo*Ciclo $\left(F(1,134)=0,45, p=0,505, \eta^{2}=0,188\right.$ para a TCM derivacional; $F(1,134)=0,60, p=0,440, \eta^{2}=0,004$ para a TCM flexional e $F(1$, $134)=0,01, p=0,908, \eta^{2}=0,000$ para o escore total). 
Uma análise descritiva observou um efeito diferenciado para as tarefas do tipo derivacional e flexional em função do sexo. Os gráficos foram colocados na mesma escala para fins de comparação. Apesar da inexistência de diferenças de sexo dentro dos subtipos das tarefas, pela Figura 2 parece haver entre estas tarefas diferentes perfis de desempenho conforme o sexo. Enquanto na tarefa derivacional no Ciclo 1 o escore aumenta levemente dos meninos para as meninas, o padrão oposto ocorre na tarefa flexional para o mesmo Ciclo. Por outro lado, parece haver uma queda de desempenho entre as meninas em relação aos meninos na tarefa derivacional no Ciclo 2, o contrário ocorrendo na tarefa flexional (escore maior para o sexo feminino).
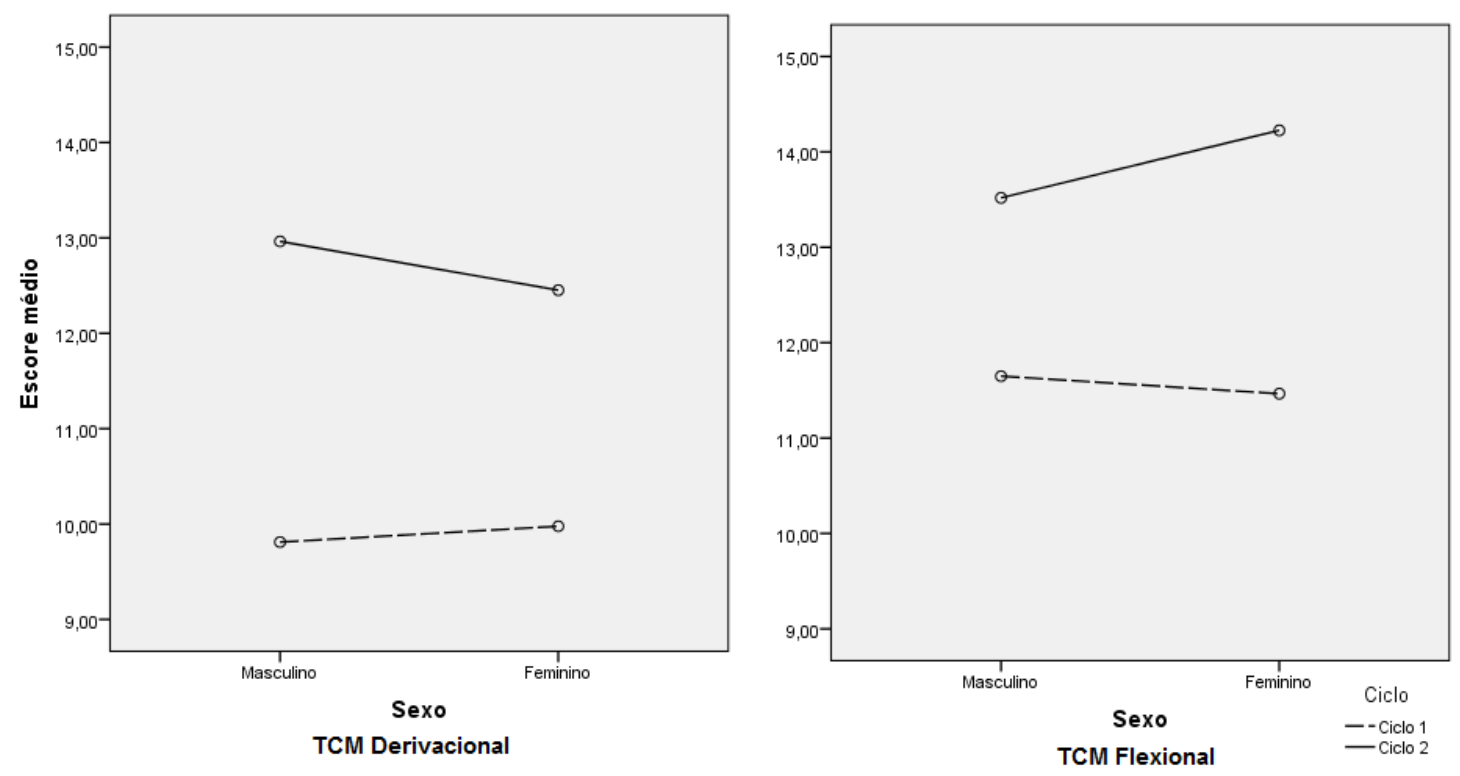

Figura 2. Comparação entre as tarefas de consciência morfológica derivacional e flexional para os sexos masculino e feminino e os Ciclos 1 e 2 .

Por fim, foi realizada uma análise de regressão linear com o intuito de evidenciar se a pontuação obtida na compreensão de leitura pode ser prevista pela pontuação da consciência morfológica. Como pré-análise, verificou-se uma correlação (Pearson) significativa entre as variáveis $(r=0,59, p<0,001)$, a qual manteve a significância mesmo após controle da idade por meio de correlação parcial $(r=0,48, p<0,001)$. A análise de regressão mostrou que o escore total na TCM previu de forma significativa o desempenho na leitura, com $R^{2}=0,296$ e $\mathrm{p}<0,001$ (i.e., a cada aumento em uma unidade no escore da TCM, aumenta-se 0,296 em compreensão leitora). Esta relação pode ser observada na Figura 3. Assim, constatou-se que $34,7 \%$ da variância da compreensão de leitura é explicada pelo desempenho na consciência morfológica ( $R^{2}$ ajustado). Esse resultado mostra que, para a amostra pesquisada, a pontuação obtida na tarefa de consciência morfológica é capaz de prever a pontuação obtida na compreensão de leitura. A mesma análise foi realizada, levando-se em consideração cada uma 
das tarefas de consciência morfológica, a saber, derivacional e flexional, e ambas contribuíram com $29 \%$ e $23 \%$, respectivamente.

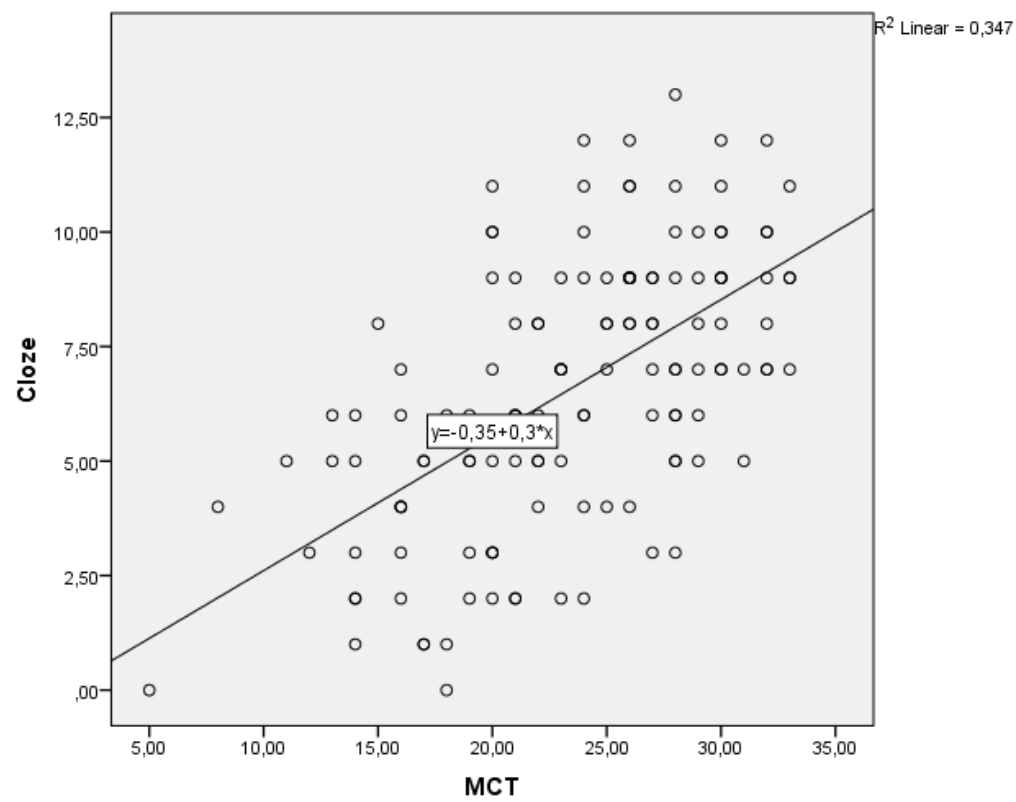

Figura 3. Scatter plot entre as pontuações da compreensão de leitura e a consciência morfológica

As análises de regressão foram repetidas, descontando-se primeiro o efeito da idade na observação da predição da TCM na compreensão leitora. Todos os resultados obtidos foram mantidos, com a TCM apresentando contribuição única para a compreensão leitora para além da idade (escore total: $\mathrm{R}^{2}=0,695 ; \mathrm{p}<$ 0,001 ; derivacional: $\mathrm{R}^{2}=0,665 ; \mathrm{p}<0,001$; flexional: $\left.\mathrm{R}^{2}=0,665 ; \mathrm{p}<0,001\right)$.

\section{DISCUSSÃO}

A partir de uma amostra de escolas de uma cidade do interior do Paraná, a presente pesquisa teve por objetivos descrever e comparar o desempenho de alunos dos Ciclos 1 e 2 do ensino fundamental de ambos os sexos em tarefas de consciência morfológica (avaliada pela TCM) e em compreensão leitora (avaliada pelo teste de Cloze). Como segundo objetivo, buscou-se investigar se a consciência morfológica é preditora da habilidade de compreensão leitora da amostra investigada. Como resultados, observou-se a presença de um efeito etário tanto em compreensão leitora quanto em consciência morfológica, indicando que estas habilidades sofrem efeito do desenvolvimento (i.e., o desempenho melhora com o decorrer da escolarização). Para a TCM, o efeito de idade se estendeu para os diferentes subtipos da tarefa (derivacional e flexional). Estes resultados estão de acordo com o encontrado pela literatura (p. ex., Diamanti et al., 2017; Guimarães et al., 2014; Law et al., 2015; Santos \& Fernandes, 2016). O desempenho dos participantes em função do sexo não se mostrou relevante para nenhuma das 
tarefas, apesar de alguns estudos apontarem que em tarefas que utilizam materiais linguísticos, em geral, as meninas apresentam melhor desempenho do que os meninos (Chiu \& McBride-Chang, 2006). Além disso, alguns estudos com adultos (Oliveira \& Santos, 2006) e crianças (Joly, Bonassi, Dias, Pioveza, \& Silva, 2014) demonstraram diferenças favoráveis para o sexo feminino no teste de Cloze. Diferenças nas análises estatísticas utilizadas entre os estudos destacados e o atual podem explicar os resultados, assim como diferenças nas faixas etárias investigadas em cada estudo.

Apesar da ausência de diferenças de sexo, uma análise descritiva mostrou perfis de desenvolvimento opostos nas tarefas de consciência derivacional e flexional em função do sexo. Enquanto que no Ciclo 1 as meninas apresentam desempenho levemente inferior aos meninos na consciência derivacional, neste mesmo ciclo o desempenho delas é levemente superior em consciência flexional. O padrão oposto ocorre no Ciclo 2, meninos com desempenho levemente superior em consciência derivacional e levemente inferior em consciência flexional. Os estudos brasileiros em consciência morfológica encontrados carecem da descrição de estatísticas descritivas por sexo e tendem a apresentar amostras com crianças muito pequenas (Mota, 2007; Mota, Anibal, et al. 2008; Mota, Gontijo, et al., 2008). Ademais, o presente estudo apresentou reduzido ' $n$ ' amostral do Ciclo 2 em relação ao Ciclo 1 , o que pode ter dificultado o aparecimento de diferenças estatísticas significativas.

Sabe-se que os indivíduos do sexo masculino apresentam uma maior frequência de diversos transtornos psiquiátricos em relação aos indivíduos do sexo feminino, inclusive problemas de aprendizagem e dislexia (p. ex., Arnett et al., 2018; Berninger, Nielsen, Abbott, Wijsman, \& Raskind, 2008). Diferenças reais entre sexos podem fornecer evidências sobre a etiologia de diversos transtornos, assim como avançar o conhecimento sobre a comorbidade. As diferenças de sexo aqui apontadas, apesar de não significativas, sinalizam a necessidade de aprimorar os estudos em consciência morfológica, a fim de investigar se trata-se de uma diferença real ou um artefato de tarefa. Novos estudos, principalmente de seguimento, devem ser realizados de modo a esclarecer se este efeito encontrado merece uma investigação mais profunda (se deve às especificidades de um delineamento transversal ou se realmente os subtipos de consciência morfológica apresentam trajetórias desenvolvimentais opostas no português brasileiro).

Conforme estudos prévios (Guimarães et al., 2014; Mota, Anibal, et al., 2008; Mota et al., 2009), houve correlação significativa entre os diferentes subtipos da consciência morfológica e o escore total na tarefa e o desempenho em compreensão da leitura. O estudo também apresentou como resultados a predição da compreensão da leitura pela consciência morfológica ( $R^{2}$ ajustado explicando entre $23 \%$ e $34 \%$ da variância em compreensão). Utilizando um delineamento longitudinal e correlacional, Barrera e Maluf (2003) demonstraram a predição da leitura pela consciência lexical e sintática. Para os processos de escrita, Guimarães 
et al. (2014) demonstraram que a consciência derivacional e flexional são significativamente preditoras da escrita graficamente correta.

Análises com a estatística alfa de Cronbach mostraram que, em geral, as tarefas apresentaram índices considerados adequados de consistência interna, tanto na amostra geral quanto por subgrupo. A exceção ocorreu principalmente no Ciclo 2, cujo resultado ficou muito abaixo da expectativa para a tarefa de compreensão e para as tarefas de consciência derivacional e flexional. Apesar disto, pelo menos no que tange à TCM, os valores ficaram semelhantes ao estudo original de validação (Mota et al., 2014). Para os outros casos, um menor tamanho amostral nesta faixa etária pode explicar os resultado. Para a tarefa de compreensão, também não se descarta o fato de o texto ser mais adequado para a amostra de crianças pequenas. Apesar destas questões, os resultados aqui obtidos mostram que, em geral, podemos ter confiança nas informações obtidas no presente estudo, principalmente para a amostra do Ciclo 1.

Finalmente, além das questões ressaltadas acima, é necessário pontuar que os resultados obtidos podem ter sido influenciados pela medida de compreensão leitora adotada no presente estudo, a saber, o teste de Cloze. Conforme ressaltado na introdução, o teste de Cloze apresenta diversos estudos apontando para sua validade como medida de compreensão leitora (p. ex., Cunha \& Santos, 2009; Fuchs et al., 1988; Greene, 2001). Entretanto, alguns autores (p. ex., Carlisle \& Rice, 2004) argumentam que o Cloze constitui uma medida relacionada à leitura, mas que não se presta a uma avaliação para além da compreensão leitora no nível da sentença (i. e., presta-se a uma avaliação da compreensão mais local e não no nível do modelo de situação, conforme descrito por Kintsch, 1988). Um estudo conduzido por Gellert e Elbro (2013) questionou esta visão, demonstrando que os processos inferenciais avaliados pelo Cloze não são necessariamente locais. Os autores utilizaram uma versão experimental do Cloze contendo múltipla escolha em cada trecho omitido e tempo limite de 10 minutos para a execução de cada texto, utilizando uma amostra de adultos e jovens adultos dinamarqueses. Como resultados, encontraram uma forte correlação (0.74) do Cloze com uma medida de compreensão baseada em respostas a perguntas sobre um texto. Apesar das divergências na literatura quanto à capacidade de o Cloze avaliar a compreensão leitora, é necessário produzir, em estudo futuros, resultados baseados em diferentes medidas de compreensão leitora a fim de verificar se o efeito encontrado no presente estudo refere-se a um efeito da tarefa ou que atinge, de uma maneira mais ampla, a compreensão leitora de modo geral.

\section{CONSIDERAÇÕES FINAIS}

O estudo mostrou um efeito do desenvolvimento em tarefas de consciência morfológica e compreensão textual, com alunos do Ciclo 1 apresentando desempenho significativamente inferior aos alunos mais velhos. Na tarefa de TCM, 
este efeito está presente tanto para o tipo derivacional quanto para o flexional, mas uma análise descritiva apontou para a possibilidade de perfis desenvolvimentais diferenciados para estas tarefas em função do sexo. Estudos com delineamento longitudinal devem ser conduzidos para esclarecer esta possibilidade. Conforme esperado, a consciência morfológica mostrou-se preditora da compreensão, o que implica que esta habilidade deve ser considerada pelos clínicos no momento de investigar dificuldades de aprendizagem, constituindo um elemento a ser introduzido na agenda para os diagnósticos dessas dificuldades. Estes resultados mantiveram-se significativos mesmo após descontar os efeitos da idade. Como limitações, a pesquisa não considerou como controles outros elementos importantes para o desempenho de compreensão leitora, tais como a leitura de palavras isoladas, a memória de trabalho e o vocabulário. Novas pesquisas devem ser conduzidas de modo a verificar como no português brasileiro um modelo inclusivo destas variáveis apontadas se estabelece. Além disso, o reduzido tamanho amostral nos anos finais não possibilitou que análises fossem feitas considerando-se os anos escolares consecutivos. Finalmente, a amostra foi composta apenas por crianças de escolas públicas, o que limita a extensão do alcance dos resultados. Pesquisas futuras devem considerar essas questões para esclarecer ainda mais as relações entre a consciência morfológica e a compreensão leitora.

\section{DECLARAÇÃO DE CONFLITO DE INTERESSES}

A primeira autora é também editora da Revista Estudos Interdisciplinares em Psicologia. Os demais autores não declaram conflitos de interesses.

\section{REFERÊNCIAS}

Anglat, H. D. (2008). El test Cloze en la evaluacion de la comprension del texto informativo de nivel universitario. Revista de Linguística Teórica y Aplicada, 46(1), 121-137.

Arnett, A. B., Cairney, B. E., Wallace, A. S., Gerdts, J., Turner, T. N., Eichler, E. E., \& Bernier, R. A. (2018). Comorbid symptoms of inattention, autism, and executive cognition in youth with putative genetic risk. Journal of Child Psychology and Psychiatry, 59(3), 268-276. doi:10.1111/jcpp.12815

Barrera, S. D., \& Maluf, M. R. (2003). Consciência metalingüística e alfabetização: Um estudo com crianças da primeira série do ensino fundamental. Psicologia: Reflexão e Crítica, 16(3), 491-502. doi:10.1590/S010279722003000300008 
Braibant, J. (1997). A decodificação e a compreensão: Dois componentes essenciais da leitura no $2^{\circ}$ ano primário. In J. Grégoire \&, B. Piérart (Eds.), Avaliação dos problemas de leitura: Os novos modelos teóricos e suas implicações diagnósticas (pp. 167-187). Porto Alegre, RS: Artes Médicas.

Berninger, V. W., Nielsen, K. H., Abbott, R. D., Wijsman, E., \& Raskind, W. (2008). Gender differences in severity of writing and reading disabilities. Journal of School Psychology, 46(2), 151-172.

Cain, K., Bryant, P., \& Oakhill, J. (2004). Children's reading comprehension ability: Concurrent prediction by working memory, verbal ability, and component skills. Journal of Educational Psychology, 96(1), 31-42.

Carlisle J. F. (2000). Awareness of the structure and meaning of morphologically complex words: Impact on reading. Reading and Writing, 12, 169-190. doi: $10.1023 / A: 100813192$

Carlisle, J., \& Rice, M. (2004). Assessment of reading comprehension. In A. Stone, E. Silliman, B. Ehren, \& K. Apel (Eds.), Handbook of language and literacy (pp. 521-555). New York, NY: Guilford.

Chiu, M. M., \& Mcbride-Chang, C. (2006). Gender, context, and reading: A comparison of students in 43 countries. Scientific Studies of Reading, 10(4), 331-362. doi: $10.1207 /$ s1532799xssr1004_1

Cunha, V. L. O., \& Capellini, S. A. (2016). Caracterização do desempenho de escolares do $3^{\circ}$ ao $5^{\circ}$ ano do ensino fundamental em compreensão de leitura. Revista CEFAC, 18(4), 941-951. doi:10.1590/1982-0216201618421215

Cunha, N. B., \& Santos, A. A. A. (2009). Validade por processo de resposta no teste de Cloze. Fractal: Revista de Psicologia, 21(3), 549-562.

Deacon, S., \& Kirby, J. (2004). Morphological awareness: Just "more phonological"? The roles of morphological and phonological awareness in reading development. Applied Psycholinguistics, 25, 223-238.

Déjean, H. (1998). Morphemes as necessary concept for structures discovery from untagged corpora. In D.M.W. Powers (Ed.), Workshop on paradigms and grounding in language learning (pp. 295-298). ACL.

Diamanti, V., Mouzaki, A., Ralli, A., Antoniou, F., Papaioannou, S., \& Protopapas, A. (2017). Preschool phonological and morphological awareness as longitudinal predictors of early reading and spelling development in Greek. Frontiers in Psychology, 8. doi:10.3389/fpsyg.2017.02039

Elliott, A. C., \& Woodward, W. A. (2007). Statistical analysis quick reference guidebook: With SPSS examples. Thousand Oak, CA: Sage.

Fuchs, L. S., Fuchs, D. \& Maxwell, L. (1988). The validity of informal reading comprehension measures. Remedial and Special Education, 9(20), 21-28. doi: $10.1177 / 074193258800900206$

Freed, E. M., Hamilton, S. T., \& Long, D. L. (2017). Comprehension in proficient readers: The nature of individual variation. Journal of Memory and Language, 97, 135-153. doi: 10.1016/j.jml.2017.07.008 
Freitas Junior, P. V. D., \& Mota, M. M. P. E. D. (2015). So, Morphological Awareness Contributes to Reading in Brazilian Portuguese?. Psico-USF,20(3), 471-480. doi:10.1590/1413-82712015200309

Ghasemi, A., \& Zahediasl, S. (2012). Normality tests for statistical analysis: a guide for non-statisticians. International journal of endocrinology and metabolism, 10(2), 486-489. doi:10.5812/ijem.3505

Gellert, A. S., \& Elbro, C. (2013). Cloze tests may be quick, but are they dirty? Development and preliminary validation of a cloze test of reading comprehension. Journal of Psychoeducational Assessment, 31(1), 16-28. doi:10.1177/0734282912451971

Greene, B. (2001). Testing reading comprehension of theoretical discourse with cloze. Journal of Research in Reading, 24(1), 82-98. doi:10.1111/14679817.00134

Guimarães, S. B., \& Mota, M. M. P. E. (2016). Qual a contribuição da consciência morfológica das crianças na precisão de leitura de palavras e compreensão de texto no português? Estudos de Psicologia, 21(3), 239-248.

Guimarães, S. R. K., Paula, F. V., Mota, M. M. P. E., \& Barbosa, V. R. (2014). Consciência morfológica: Que papel exerce no desempenho ortográfico e na compreensão de leitura? Psicologia USP, 25(2), 201-212. doi:10.1590/01036564A20133713

Harris, Z. S. (1955). From morpheme to phoneme. Language, 31(2), 190-222.

Ho, C.S.H., \& Bryant, P. (1997). Learning to read Chinese beyond the logographic phase. Reading Research Quarterly, 32, 276-289.

Joly, M. C. R., Bonassi, J., Dias, A. S., Pioveza, N. M., \& Silva, D. V. (2014). Avaliação da compreensão de leitura pelo Sistema Orientado de Cloze (SOC). Fractal: Revista de Psicologia, 26(1), 223-242. doi:10.1590/S198402922014000100016

Keshavarz, M. H, \& Salimi, H. (2007). Collocational competence and cloze test performance: A study of Iranian EFL learners. International Journal of Applied Linguistics, 17(1), 81-92.

Kintsch, W. (1988). The role of knowledge in discourse comprehension: A construction-integration model. Psychological Review, 95(2), 163-182. doi:10.1037/0033-295X.95.2.163

Kobayashi, M. (2002). Cloze tests revisited: Exploring item characteristics with special attention to scoring methods. Modern Language Journal, 86(4) 571586. doi:10.1111/1540-4781.00162

Law, J. M., Wouters, J., \& Ghesquière, P. (2015). Morphological awareness and its role in compensation in adults with dyslexia. Dyslexia, 21(3), 254-272. doi: 10.1002 /dys. 1495

Lencastre, L. (2003). Leitura: A compreensão de textos. Lisboa: Fundação Calouste Gulbenkian. Ministério da Ciência e do Ensino Superior. 
Li, H., Dronjic, V., Chen, X., Li, Y., Cheng, Y., \& Wu, X. (2017). Morphological awareness as a function of semantics, phonology, and orthography and as a predictor of reading comprehension in Chinese. Journal of child language, 44(5), 1218-1247. doi:10.1017/S0305000916000477

Li, W., Anderson, R. C., Nagy, W., \& Zhang, H. (2002). Facets of metalinguistic awareness that contribute to Chinese literacy. In W. Li, J. S. Gaffney, \& J. L., Packard (Eds.), Chinese children's reading acquisition: Theoretical and pedagogical issues (pp. 59-86). Norwell, MA: Kluwer.

Liu, Y., Sun, H., Lin, D., Li, H., Yeung, S. S. S., \& Wong, T. T. Y. (2018). The unique role of executive function skills in predicting Hong Kong kindergarteners' reading comprehension. British Journal of Educational Psychology. Advanced online publication. doi:10.1111/bjep.12207

Lyster, S. A. H., Lervåg, A. O., \& Hulme, C. (2016). Preschool morphological training produces long-term improvements in reading comprehension. Reading and writing, 29(6), 1269-1288. doi: $10.1007 / \mathrm{s} 11145-016-9636-x$

Manolitsis, G., Grigorakis, I., \& Georgiou, G. K. (2017). The longitudinal contribution of early morphological awareness skills to reading fluency and comprehension in Greek. Frontiers in psychology, 8, 1793. doi: $10.3389 /$ fpsyg.2017.01793

Mckamey, T. (2006). Getting closure on cloze: A validation study of the "rational deletion" method. Second Language Studies, 24(2), 114-164.

Mota, M. M. P. E. (2007). Complexidade fonológica e reconhecimento da relação morfológica entre as palavras: Um estudo exploratório. Revista da Vetor Editora, 8(2), 131-138.

Mota, M. M. P. E. (2009). O papel da consciência morfológica para a alfabetização em leitura. Psicologia em Estudo, 14(1), 159-166. doi:10.1590/S141373722009000100019

Mota, M. M. P. E., Anibal, L. \& Lima, S. (2008). A Morfologia Derivacional Contribui para a Leitura e Escrita no Português? Psicologia: Reflexão e Crítica, 21(2), 311-318. doi:10.1590/S0102-79722008000200017

Mota, M. M. P. E., Gontijo, R., Mansur-Lisboa, S., Olive, R., Silva, D., ... \& Kamisaki, R. (2008). Avaliação da consciência da morfologia derivacional: Fidedignidade e validade. Avaliação Psicológica, 7(2), 151-157.

Mota, M. M. P. E., Lisboa, R., Dias, J., Gontijo, R., Paiva, N., ..., Silva, D. A., \& Santos, A. A. A. (2009). Relação entre consciência morfológica e leitura contextual medida pelo teste de Close. Psicologia: Reflexão e Crítica, 22(2), 223-229. doi:10.1590/ S0102-79722009000200008

Mota, M. M. P. E., Mota, D., Cotta, J., Lima, S. A., Calzavara, A., Cunha, N., \& Banhato, E. (2009). Consciência morfossintática, alfabetização e contextos do desenvolvimento. Psico-USF, 14(1), 11-18. doi.10.1590/S141382712009000100003 
Mota, M. M. P. E., Santos, A. A. A., \& Guimarães, S. B. (2014). Evidências de validade e consistência interna de tarefas de analogia gramatical. Estudos de Psicologia, 19(4), 250-277.

Nagy, W., Berninger, V., \& Abbot, R. (2006). Contributions of morphology beyond phonology to literacy outcome of upper elementary and middle-school students. Journal of Educational Psychology, 98(1), 134-147.

Nouwens, S., Groen, M. A., \& Verhoeven, L. (2017). How working memory relates to children's reading comprehension: The importance of domain-specificity in storage and processing. Reading and writing, 30(1), 105-120. doi:10.1007/s11145-016-9665-5

Nunes, T., Bindman, M., \& Bryant, P. (1997). Morphological strategies: Developmental stages and processes. Developmental Psychology, 33(4), 637-649. doi:10.1037//0012-1649.33.4.637

Oliveira, K. L., \& Santos, A. A. A. (2006). Compreensão de textos e desempenho acadêmico. Psic: Revista da Vetor Editora, 7(1), 19-27.

Pallant, J. (2010). SPSS survival manual: A step by step guide to data analysis using SPSS. Maidenhead: Open University Press/McGrawHill.

Santos, A. A. A. (2005). O Teste de Cloze como instrumento de avaliação da compreensão em leitura. Relatório técnico. Itatiba: Universidade São Francisco.

Santos, A. A. A., \& Fernandes, E. S. O. (2016). Habilidade de escrita e compreensão de leitura como preditores de desempenho escolar. Psicologia Escolar e Educacional, 20(3), 465-473. doi.10.1590/21753539/2015/02031013

Santos, A. A. A., \& de Oliveira, E. Z. (2010). Avaliação e desenvolvimento da compreensão em leitura no ensino fundamental. Psico-USF, 15(1), 81-91. doi:10.1590/S1413-82712010000100009

Soares, M. B. (1995). Língua escrita, sociedade e cultura: Relações, dimensões e perspectivas. Revista Brasileira de Educação, (0), 5-16.

Spinillo, A., Mota, M. M. P. E., \& Correa, J. (2010). Consciência metalinguística e compreensão de leitura: Diferentes facetas de uma relação complexa. Educar em Revista, 38, 157-171. doi:10.1590/S0104-40602010000300011

Suehiro, A. C. B., \& Santos, A. A. A. (2015). Compreensão de leitura e consciência fonológica: Evidências de validade de suas medidas. Estudos de Psicologia (Campinas), 32(2), 201-211. doi:10.1590/0103-166X2015000200005

Tan, Y., Martin, R. C., \& Van Dyke, J. A. (2017). Semantic and Syntactic Interference in Sentence Comprehension: A Comparison of Working Memory Models. Frontiers in psychology, 8, 198.c doi: 10.3389/fpsyg.2017.00198

Taylor, G. (1953). Dispersion of soluble matter in solvent flowing slowly through a tube. Proceedings of the Royal Society A: Mathematical, Physical and Engineering Sciences, 219(1137), 186-203. doi:10.1098/rspa.1953.0139 
Tighe, E. L., \& Schatschneider, C. (2016a). Examining the relationships of component reading skills to reading comprehension in struggling adult readers: A meta-analysis. Journal of Learning Disabilities, 49(4), 395-409. doi:10.1177/0022219414555415

Tighe, E. L., \& Schatschneider, C. (2016b). A quantile regression approach to understanding the relations among morphological awareness, vocabulary, and reading comprehension in adult basic education students. Journal of Learning Disabilities, 49(4), 424-436. doi: 10.1177/0022219414556771

Tong, X., Deacon, S., Parrila, S., \& Kirby, J. (2011). Morphological awareness: A key to understanding poor reading comprehension in English. Journal of Educational Psychological Association, 103(3), 523-534.

Urbina, S. (2014). Essentials of psychological testing. Hoboken, New Jersey: John Wiley \& Sons.

Yuill, N. M., \& Oakhill, J. V. (1996). Higher order factors in comprehension disability: Processes and remediation. In C. Cornoldi, \& J. V Oakhill (Eds.), Reading difficulties: Processes and intervention (pp. 69-92). Mahwah, NJ: Lawrence Erlbaum. 
Sobre os autores

Patrícia Silva Lúcio é psicólogo pela Universidade Federal de Minas Gerais, mestre pela mesma universidade e doutora pelo programa de Pós-Graduação em Psiquiatria e Psicologia Médica pela Universidade Federal de São Paulo.

Atualmente é pós-doutoranda em Psiquiatria pela Universidade de São Paulo. Professora Adjunta da Universidade Estadual de Londrina. pslucio@gmail.com Thatiana Helena de Lima é psicóloga, mestre e doutora pela Universidade São Francisco. Professora Adjunta da Universidade Federal da Bahia. thatianahlima@gmail.com

Ana Deyvis Santos Araújo Jesuíno é psicóloga pela FACID, mestre e doutoranda Universidade São Francisco. Bolsista CAPES. anadeyvis@gmail.com

Fabián J. M. Rueda é psicólogo, mestre e doutor pela Universidade São Francisco. Professor e coordenador do Programa de Pós-Graduação Stricto Sensu em Psicologia da Universidade São Francisco. É Bolsista de Produtividade em Pesquisa do CNPq - Nível 1D. marinfabian@gmail.com

A contribuição de cada autor pode ser atribuída como se segue: P.S.L e T.H.L. trabalharam na conceituação, redação, análise e interpretação dos dados e revisão do manuscrito. A.D.S.A.J e F.J.M.R. trabalharam na redação, interpretação dos dados e revisão do manuscrito. P.S.L. trabalha em projeto que parcialmente financiou a pesquisa.

Os autores agradecem à Fundação Araucária pelo financiamento da pesquisa.

Recebido em: 16/10/2017

Revisado em: 06/03/2018

Aceito em: $10 / 03 / 2018$ 